\title{
Attitude Reorientation of Spacecraft with Attitude Forbidden Zones
}

\author{
Xuhui Lu \\ The Seventh Research Division and the Department of Systems and Control, Beihang University (BUAA), 37 XueYuan \\ Road, Beijing, 100191, P.R. China \\ Yingmin Jia \\ The Seventh Research Division and the Department of Systems and Control, Beihang University (BUAA), 37 XueYuan \\ Road, Beijing, 100191, P.R. China \\ E-mail: luxuhuiluxuhui@163.com,ymjia@buaa.edu.cn \\ www.buaa.edu.cn
}

\begin{abstract}
This paper investigates an attitude reorientation control scheme of spacecraft, considering attitude forbidden zone and external disturbances. A novel potential barrier function is proposed with an attitude constraint term and a dynamical scaling term. Here the dynamical scaling term is synthesized with a backstepping-based control scheme to accommodate both the avoidance of attitude forbidden zones and disturbance attenuation. Simulation results are given to verify the effectiveness of the proposed method.
\end{abstract}

Keywords: attitude stabilization, attitude constraints, backstepping, dynamic scaling

\section{Introduction}

The attitude reorientation of spacecraft has been extensively studied in the past decades. However, during large-angle attitude maneuvering of spacecraft, some sensitive on-board instruments such as infrared telescopes and interferometers should avoid direct exposure of bright objects like the sun. Therefore, the spacecraft reorientation with the attitude forbidden zones, or the constrained attitude control problem (CAC), is a topic of great interest in applications. In the early work, ${ }^{1}$ via implicit magnitude constraint of quaternion, the non-convex attitude constraints can be transformed into linear matrix inequalities (LMIs) and this constrained optimization problem can be analyzed in the semi-definite programming framework. Then, this convex parameterization can be even used in the construction of logarithmic barrier function to derive controller. $^{2}$ Other methods, such as invariant set, ${ }^{3}$ randomized algorithm (RA), ${ }^{4}$ etc., have also been applied to solve the problem.

However the spacecraft is not free from disturbances, which are not considered in the above methods. Those disturbances are from, e.g., the external environment like atmosphere drag, and the attitude reorientation property would be reduced and even the avoidance of attitude constraint zone is not ensured if disturbances are neglected. Thus a fault-tolerant sliding-mode control scheme is derived in Ref. 5 to accommodate disturbances and actuator fault, where exact upper bound of uncertain inertia matrix can be online estimated. Besides, a finite-time output feedback control scheme is derived in Ref. 6 for spacecraft stabilization with bounded disturbances.

The aim of the paper is to provide a controller to deal with spacecraft repointing problem in the presence of attitude forbidden zone and disturbances. The main 
contributions of this paper, compared with the previous results, is the construction of a novel attitude barrier potential function, where the unwanted term in the backstepping controller design is compensated by a scaling factor. Therefore both the avoidance of attitude forbidden zones and disturbance attenuation are achieved.

The remainder of the paper is organized as follows. Section 2 lists the preliminaries including the modeling of spacecraft, attitude forbidden zones and control objective. Controller design with the corresponding proof is given in Section 3. Simulation results and conclusions are offered in Section $4 \& 5$.

\section{Preliminaries}

The dynamics of spacecraft is listed below [6]

$$
\begin{gathered}
\dot{q}=L(q) w \\
J \dot{w}+S(w) J w=u+d
\end{gathered}
$$

where, for vectors in (1)(2), $q=\left[q_{0} ; q_{v}\right] \in R^{4}$ is quaternion with $q^{T} q=1, w \in R^{3}$ angular velocity of spacecraft in the body-attached frame, $u \in R^{3}$ control torque from actuators of spacecraft, and $d \in R^{3}$ external disturbances. Moreover, for the matrices in (1) and (2) $L(q)=\left[-q_{v} ; q_{0} I_{3}+S\left(q_{v}\right)\right]$ where $S(a)=\left[0,-a_{3}, a_{2} ; a_{3}\right.$, $\left.0,-a_{1} ;-a_{2}, a_{1}, 0\right]$ with $a \in R^{3}$, and $J \in R^{3 \times 3}$ is inertia matrix with $J>0$.

In practice, when spacecraft is rotated towards specific orientation, some sensitive on-board instruments like interferometer should circumvent direct exposure of bright celestial objects such as the sun. These unwanted orientations are considered as attitude forbidden zones, and it can be described $a^{2}$

$$
x^{T} R y<\cos \theta
$$

where $R=\left(q_{0}^{2}-q_{v}^{T} q^{T}\right) I_{3}+2 q_{v} q_{v}^{T}+2 q_{0} S\left(q_{v}\right)$ is rotation matrix of spacecraft from body-attached frame to inertia frame, $x$ is the direction of bright and celestial object in the inertia frame, $y$ is the instrument's bore-sight vector in the body-attached frame, $R y$ is the counterpart in the inertia frame, $\beta$ is the angle between $x$ and $R y$, and $\theta \in(0, \pi)$ is the minimum angle allowed for $\beta$. Note that $x$ and $y$ are constant. into $^{2}$

Furthermore the above constraint can be transformed

$$
q^{T} M(x, y, \theta) q<0
$$

where $M(x, y, \theta)=\left[A, b ; b^{T}, d\right]$ with $A=x y^{T}+y x^{T}-$ $\left(x^{T} y+\cos \theta\right) I_{3}, b=x \times y, d=x^{T} y-\cos \theta$. Moreover, the inequality (4) can be written in more accurate form $-1-\cos \theta \leq q^{T} M(x, y, \theta) q<0$.

It is noted that $q^{T} M q \rightarrow 0$ if $\beta \rightarrow \theta$. The property facilitates the construction of barrier potential function. For simplicity, only one bore-sight vector and one attitude forbidden zone are considered here.

The control objective is to render the spacecraft rotate towards the prescribed attitude $q_{d}$ or $-q_{d}$, both of which is equivalent in the physical meaning, from any permissible initial value of attitude $q(0)$ and angular velocity $w(0)$. During the process the spacecraft circumvent the attitude forbidden zones $\Xi_{q}=\left\{q \in S^{3}\right.$ $\left.\mid q^{T} M q<0\right\}$ in the presence of external disturbances. It is obvious that $q_{d}, q(0)$ are outside attitude forbidden zone, i.e. $q_{d}, q(0) \nsubseteq \Xi_{q}$. Moreover in the design, there exist $J_{\text {min }}>0, J_{\text {max }}>0$ such that $J_{\text {min }} I_{3}<J<J_{\max } I_{3}$, and disturbances $d(t)$ are bounded.

\section{Controller Design}

Compared with the barrier potential function in Ref. 1 and 2, the barrier function of quaternion here is modified to accommodate external disturbances.

First consider quaternion dynamics (1). The corresponding Lyapunov-like barrier function is derived to be $V_{q}=\left(k_{1} / r_{q}+k_{2} \ln \left(-2 / q^{T} M q\right)\right)\left\|q-q_{d}\right\|^{2}$ where the positive variable $r_{q}$ is a scaling factor that would be defined later, and $k_{1}>0, k_{2}>0$. Here the virtual control $w_{c}$ for $w$ is set as $w_{c}=k_{3} L^{T}(q) q_{d}$ with $k_{3}=\bar{k}_{3} / r_{q}$ and $\bar{k}_{\underline{3}}>0$, and the dynamics of $r_{q}$ is

$$
\begin{aligned}
\dot{r}_{q} & =-\frac{k_{2} \bar{k}_{3}}{8 k_{1}}\left(r_{q}-r_{q c}\right) \ln \left(-\frac{2}{q^{T} M q}\right)\left(1+q_{d}^{T} q\right) \\
& +\operatorname{Proj}_{r_{q}}\left(\frac{2 k_{2} \bar{k}_{3} r_{q}}{k_{1} q^{T} M q} q^{T} \operatorname{ML}(q) L^{T}(q) q_{d}\right)
\end{aligned}
$$

where $\operatorname{Proj}_{r_{q}}(\cdot)$ here is defined in Ref 7, with $r_{q c}>0$. Thus after a lengthy calculation, the derivative of $V_{q}$ is simplified based on (1) (5)as 


$$
\begin{gathered}
\dot{V}_{q} \leq-\left(\frac{k_{1}}{r_{q}}-k_{2} \ln \left(-\frac{q^{T} M q}{2}\right)\right) 2 q_{d}^{T} L(q) w_{r} \\
-\frac{4 k_{2}\left(1-q_{d}^{T} q\right)}{q^{T} M q} q^{T} M L(q) w_{r} \\
-\frac{k_{3}}{2}\left(\frac{k_{1}}{r_{q}}-\frac{k_{2}}{2} \ln \left(-\frac{q^{T} M q}{2}\right)\right)\left(1-\left(q_{d}^{T} q\right)^{2}\right)
\end{gathered}
$$

with $\left\|q-q_{d}\right\|^{2}=2\left(1-q_{d}^{T} q\right), q_{d}^{T} L(q) L^{T}(q) q_{d}=\frac{1}{4}(1-$ $\left.\left(q_{d}^{T} q\right)^{2}\right)$ used. Here it should be also noted that $r_{q} \geq r_{q c}$ given $r_{q}(0) \geq r_{q c}$.

Besides the dynamics of $w$ is considered. Take the derivative of $w_{r}$ and from (2) we get

$$
J \dot{w}_{r}=-S(w) J w+u+d-J \dot{w}_{c}
$$

with $\dot{w}_{c}=-k_{3} L^{T}\left(q_{d}\right) L(q) w+k_{3}\left(\dot{r}_{q} / r_{q}\right) L^{T}\left(q_{d}\right) q$. Let $V_{w_{r}}$ $=w_{r}^{T} J w_{r} / 2$ and accordingly $u=S\left(w_{c}\right) J w+J_{0} \dot{w}_{c}+u_{1}$, thus based on (7) the derivative of $V_{w_{r}}$ is

$$
\dot{V}_{w_{r}}=w_{r}^{T}\left\{u_{1}+d\right\}
$$

Then $V_{q}$ and $V_{w_{r}}$ is combined together and therefore set $V_{c}=k_{4} V_{q}+V_{w_{r}}$ with $k_{4}>0$. Here $u_{1}$ is set as

$$
\begin{aligned}
& u_{1}=2 k_{4}\left(\frac{k_{1}}{r_{q}}-k_{2} \ln \left(-\frac{q^{T} M q}{2}\right)\right) L^{T}(q) q_{d} \\
& +\frac{4 k_{2} k_{4}}{q^{T} M q}\left(1-q_{d}^{T} q\right) L^{T}(q) M q-\left(k_{5}+k_{6}\right) w_{r},
\end{aligned}
$$

with $k_{5}>0, k_{6}>0$. Therefore based on (6)(8)(9) the derivative of $V_{c}$ can be scaled as

$$
\dot{V}_{c} \leq-\Pi_{2}+\Theta_{1}
$$

where

$$
\Pi_{2}=\frac{k_{3} k_{4}}{2}\left(k_{2}-\frac{1}{2 r_{q}} \ln \left(-\frac{q^{T} M q}{2}\right)\right)\left(1-\left(q_{d}^{T} q\right)^{2}\right)+k_{6} w_{r}^{T} w_{r}
$$
and $\Theta_{1}=\bar{d}^{T} \bar{d} / 4 k_{5}$, and young inequality is used here.

Here the total control torque $u$ is summarized as

$$
\begin{gathered}
u=S\left(w_{c}\right) J w+2 k_{4}\left(\frac{k_{1}}{r_{q}}-k_{2} \ln \left(-\frac{q^{T} M q}{2}\right)\right) L^{T}(q) q_{d} \\
+J_{0} \dot{w}_{c}+\frac{4 k_{2} k_{4}}{q^{T} M q}\left(1-q_{d}^{T} q\right) L^{T}(q) M q-\left(k_{5}+k_{6}\right) w_{r}
\end{gathered}
$$

Before the main result is preceded, it should be noted that both the stability of the variables in Lyapunov function $V_{\bar{t}}$ and the boundedness of $r_{q}$ should be considered, both of which lead to Theorem 1 .
Theorem 1. Consider system (1)(2) and controller (5) (11). For $\forall q(0)$ with $q^{T}(0) q(0)=1, q(0) \nsubseteq \Xi_{q}, \forall w(0)$, and $r_{q}(0) \geq r_{q c}$, the following properties hold:

(i). The variables $q, w$ are bounded for $\forall t \geq 0$, and $q^{T}(\mathrm{t}) M q(\mathrm{t})<0$, meaning that spacecraft would not rotate into attitude forbidden zone all the time.

(ii). The variables $q, w$ would be within a small neighbor of desired equilibrium points $\left( \pm q_{d}, 0_{3}\right)$.

(iii). The scaling factor $r_{q}(t)$ is bounded all the time with the appropriate parameters in the controller and a switching strategy of desired quaternion from $q_{d}$ to $-q_{d}$ when $q_{d}^{T} q \leq-1+\varepsilon$ where $\varepsilon \in(0,1]$.

Proof. First from (10), it is concluded that $V_{\bar{t}}$ is bounded all the time, and besides $q^{T} M q<0$ all the time.

For the second property, $\Theta_{2}$ can be set as sufficiently small as possible via $k_{5}, k_{6}, \Gamma_{\theta}$. Thus like ${ }^{[6]}, V_{\bar{t}}$ would converge into a neighbor of origin, as sufficient close as possible, in finite time and property 2 is ensured. Moreover, based on properties 1 and 2, there exist $\varphi>0$ such that $q^{T} M q<-\varphi$, leading to forward completeness of $r_{q}$.

As for the third property, via appropriate parameters, there exists $\bar{M}>0$ for $r_{q}$ such that $\|\left(2 k_{2} \bar{k}_{3} r_{q}\right) /\left(k_{1} q^{T} M q\right)$ $q^{T} M L(q) \|<\bar{M} r_{q}$. Besides, $\left(k_{2} \bar{k}_{3}\right) /\left(8 k_{1}\right) \ln \left(-2 / q^{T} M q\right)$ $\left(1+q_{d}^{T} q\right) \geq \bar{k}_{q} \triangleq\left(k_{2} \bar{k}_{3}\right) /\left(8 k_{1}\right) \ln (2 /(1+\cos \theta)) \varepsilon \quad$ based on $-1-\cos \theta \leq q^{T} M q$ and switching strategy of desired quaternion $q_{d}$. Thus $\dot{r}_{q} \leq-\bar{k}_{q}\left(r_{q}-r_{q c}\right)+\bar{M}\left\|L^{T}(q) q_{d}\right\| r_{q}$. Moreover based on property 2, there exist $\epsilon_{1}>0$, where $\epsilon_{1} \leq \bar{k}_{q} /(2 \bar{M})$, and the corresponding $T_{1}>0$ such that $\left\|L^{T}(q) q_{d}\right\|<\epsilon_{1}, \forall t \geq T_{1}$. Therefore it is concluded that $\dot{r}_{q} \leq 0$ when $r_{q} \geq 2 r_{q c}, t \geq T_{1}$, which means $r_{q}$ is bounded when $t \geq T_{1}$. Besides $r_{q}$ is also bounded when $0 \leq t \leq T$ due to boundedness of $q$ and $q^{T} M q<-\varphi$. In all, $r_{q}$ is bounded all the time and the proof is complete.

\section{Simulation Results}

The controller scheme is simulated to verify its effectiveness. In the simulation, $J=[350,3,4$; $3,270,10 ; 4,10,190]$ with $J_{\text {max }}=600, J_{\text {min }}=100$. As for attitude forbidden zone, $x=[0.35 ; 0.24 ; 0.9055]$, $y=[1 ; 0 ; 0], \theta=60^{\circ}$. Moreover initial values are set as 
$q(0)=[0.5,-0.5,-0.5,-0.5]^{T}, \quad w(0)=[0.1,-0.2,0.12]^{T}$, $r_{q}(0)=1$, and control parameters are $k_{1}=2, k_{2}=4$, $k_{3}=0.6, k_{4}=4, k_{5}=0.4, k_{6}=25, r_{q c}=0.5$.Moreover in the simulation, the control torque $u$ is bounded, i.e., $\left|u_{i}\right|<10 N \cdot m, i=1,2,3$, and external disturbances are set as $d=\left[0.5 \sin \left(0.2 t-45^{\circ}\right) ; 0.6 \sin \left(0.2 t+30^{\circ}\right)\right.$; $0.3 \cos (0.2 t)]$.

It can be seen from Fig. 1 that the spacecraft is initially located where the bore-sight vector is close to the attitude forbidden zone. Here the controller renders spacecraft circumvent the attitude forbidden zone, and eventually stop at the desired attitude, where the boresight vector is also in proximity to attitude forbidden zone. Besides disturbances are attenuated during the process.
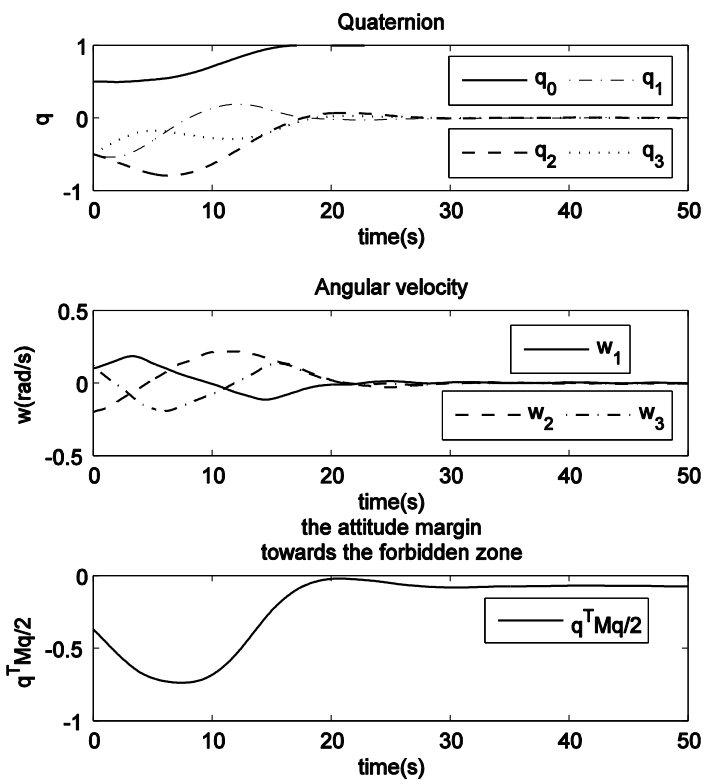

Fig.1, simulation of result of the proposed controller.

\section{Conclusion}

A Lyapunov-based control scheme for attitude constrained reorientation of spacecraft with disturbances is derived. A novel attitude potential function with a scaling factor is derived, and a corresponding controller is designed to accomplish the avoidance of attitude forbidden zone and disturbance attenuation. Future work would focus on the constrained attitude control law without measurements of angular velocity.

\section{Acknowledgements}

This work is supported by the National Basic Re-search Program of China (973 Program: 2012CB821200, 2012CB821201) and the NSFC (61134005, 60921001, $61327807)$.

\section{References}

1. Y. Kim, M. Mesbahi (2004), Quadratically constrained attitude control via semidefinite programming, IEEE Transaction on Automatic Control, 49(5) :731-735.

2. U. Lee, M. Mesbahi (2012), "Spacecraft Reorientation in Presence of Attitude Constraints via Logarithmic Barrier Potentials", in Proceedings of American Control Conference, San Francisco, CA, 450-455.

3. A. Weiss, F. Leve, M. Baldwin, et al., (2014), "Spacecraft Constrained Attitude Control using Positively Invariant Constraint Admissible Sets on $S O(3) \times R_{3}$ ", in Proceeding of American Control Conference, Portland, Oregon, 4955-4960.

4. P. Y. Cui, W. G. Zhong, H. T. Cui (2007), "Onboard spacecraft slew-planning by heuristic state spacecraft search and optimization", in Preceding of the 2007 IEEE International Conference on Mechatronics and Automation, Harbin, China, 2115-2119.

5. Q. L. Hu (2010), Robust adaptive sliding-mode faulttolerant control with $L_{2}$-gain performance for flexible spacecraft using redundant reaction wheels, IET Control Theory and Applications, 4(6): 1055-1070.

6. A. M. Zou (2009), Finite-time output feedback attitude tracking control for rigid spacecraft, American Control Conference, IEEE Transactions on Control System Technology, 22(1), 338-345.

7. Ø. N. Stamnes, O. M. Aamo, G. O. Kaasa (2011), A constructive speed observer design for general EulerLagrange systems, Automatica, 47(10), pp. 2233-2238. 